\title{
Heat Transfer Analysis of a Rectangular Moving Porous Fin with Temperature-Dependent Thermal Conductivity and Internal Heat Generation: Comparative and Parametric Studies
}

\author{
M. G. Sobamowo*, A. A. Yinusa, M. O. Salami, O. C. Osih, B. O. Adesoye \\ Department of Mechanical Engineering, University of Lagos, Akoka, Lagos State, Nigeria.
}

\begin{abstract}
How to cite this paper: M. G. Sobamowo, A. A. Yinusa, M. O. Salami, O. C. Osih, B. O. Adesoye. (2021) Heat Transfer Analysis of a Rectangular Moving Porous Fin with Temperature-Dependent Thermal Conductivity and Internal Heat Generation: Comparative and Parametric Studies. Engineering Advances, 1(2), 50-66. DOI: 10.26855/ea.2021.12.004
\end{abstract}

Received: November 11, 2021

Accepted: December 8, 2021

Published: December 29, 2021

*Corresponding author: M. G. Sobamowo, Department of Mechanical Engineering, University of Lagos, Akoka, Lagos State, Nigeria.

Email: mikegbeminiyiprof@yahoo.com

\begin{abstract}
In this work, heat transfer study of rectangular moving porous fin with temperature-dependent thermal conductivity and internal heat generation is presented using differential transformation and finite difference methods. The results of the two methods are compared and very good agreements are established. However, with the aid of the approximate analytical solution, parametric studies of the effects of thermal-geometric and thermo-physical fin parameters such as the Peclet number, thermal conductivity parameter, convection parameter, porosity parameter and internal heat generation parameter on the temperature distribution, rate of heat transfer and thermal efficiency of the fin are investigated. From the analysis, it is found that increase in porosity, convective, increase the rate of heat transfer from the fin and consequently improve the efficiency of the fin while increase in thermal conductivity and internal heat generation decreases the rate of heat transfer from the fin. It is hoped that the numerical and semi-analytical studies presented in this paper will help in providing good physical insights in practice.
\end{abstract}

\section{Keywords}

Thermal analysis, Porous Fin, Convective fin, Moving fin, Variable thermal conductivity, Differential transformation method, Finite difference Method

\section{Introduction}

The passive augmentation of heat transfer in a thermal system is commonly achieved by the use of fins and spines. Adding a fin to an object increases the surface area of the object exposed to the surroundings and hence facilitates the rate of heat transfer. Application of fins can be found in various industrial systems such as the cooling of computer processors, air conditioning and oil carrying pipelines. In recent years, there has been extensive research on moving continuous surface. This phenomenon of a moving continuous surface occurs in a good number of industrial applications such in extrusion, hot rolling, glass fiber drawing and casting. Also in industrial processes, control of cooling rate of the sheets is very important to obtain desired material structure. Several studies were performed on heat transfer using fins and moving continuous surface [1-24]. The book by Kraus, Aziz, and Welty [1] provides comprehensive coverage of the various facets of this technology. Sobamowo [2] used the Galerkin's Method of Weighted Residual for a Convective Straight Fin with Temperature-Dependent Conductivity and Internal Heat Generation to investigate the effects of thermo-geometric parameters, the coefficient of heat transfer and thermal conductivity (non-linear) parameters on the temperature distribution, heat transfer and thermal performance of the longitudinal rectangular fin. Chiu and Chen [2] utilized the Adomian decomposition method to evaluate the efficiency and optimal length of the convective rectangular fin with variable thermal conductivity. The Adomian decomposition method was presented by Arslanturk [3] to evaluate 
the temperature distribution within the fins and also evaluated the fin efficiency. Rajabi [4] studied the efficiency of straight fins with temperature-dependent thermal conductivity using the homotopy perturbation method. A series form analytical solution using the homotopy analysis method was presented for evaluating the fin efficiency of straight convective fins by Domairry and Fazeli [5]. Kulkarni and Joglekar [6] implemented a numerical technique based on residue minimization to solve the nonlinear differential equation governing the temperature distribution in straight-convective fins having temperature-dependent thermal conductivity and further evaluated the fin efficiency. In Bouaziz and Aziz [7], the efficiency of a double optimal linearization method was compared with the homotopy perturbation method, variational method, and double series regular perturbation method in evaluating the temperature distribution in straight convective-radiative fins with variable thermal conductivity. In the work of Ranjan Das [8], a simplex search method was used to evaluate the temperature field for a conductive-convective fin with variable thermal conductivity. Aziz and Torabi [9] studied numerically the thermal performance of a convective-radiative fin with temperature-dependent thermal conductivity, heat transfer coefficient, and surface emissivity. Aziz and Khani [10] presented the homotopy analysis method for the analytic solution of heat transfer in moving fins with variable thermal conductivity which is losing heat to the surroundings simultaneously through convection and radiation. A numerical study of the heat process in a continuously moving rod undergoing thermal processing of variable thermal conductivity losing heat through both convection and radiation was studied by Aziz and Lopez [11]. The differential transformation method was applied by Torabi et al. [12] for analyzing the heat transfer in moving fins with temperature-dependent thermal conductivity, losing heat through both convection and radiation. Ravi and N. Uday [13] numerically investigated the heat transfer in a continuously moving convective-radiative fin with variable thermal conductivity by using Haar wavelets. In their work, they studied the effects of the significant parameters, i.e., the thermal conductivity parameter, convection-sink temperature, radiation-sink temperature, convection-conduction parameter, radiation-conduction parameter, and Peclet number, on the temperature distribution and heat transfer characteristics of a continuously moving convective-radiative fin with variable thermal conductivity.

In finding solution to nonlinear equations, the determination of Adomian polynomials as carried out in ADM, the restrictions of HPM to weakly nonlinear problems, the lack of rigorous theories or proper guidance for choosing initial approximation, auxiliary linear operators, auxiliary functions, and auxiliary parameters in HAM, determination of Lagrangian multiplier or parameter as carried out in VIM, operational restrictions to small domains most perturbation methods necessitated the use of another relatively simple and straight forward method for the nonlinear problem. Moreover, there viewed studies show that the application of differential transformation method has been limited to thermal analysis of solid fin under non-magnetic environment. Such application of differential transform method as introduced by Zhou [25] to solve nonlinear problems has fast gained ground as it appeared in many engineering and scientific research papers because of comparative advantages over the other approximate analytical methods. It solves nonlinear integral and differential equations without linearization, discretization or restrictive assumptions, perturbation and this is the main benefit of this method. Using DTM, a closed form series solution or approximate solution can be obtained as it provides excellent approximations to the solution of non-linear equation with high accuracy. It is a more convenient method for engineering calculations compare with other approximate analytical or numerical method. It appears more appealing than the numerical solution as it helps to reduce the computation costs, simulations and task in the analysis of nonlinear problems. Several analyses have been derived using the differential transform method (DTM). Moradi and Rafiee [26], carried out the analytical solution to convection-radiation of a continuously moving fin with temperature-dependent thermal conductivity using DTM, the differential transformation method was applied to solve simultaneous convection and radiation heat transfer problem in a continuously moving fin with temperature thermal conductivity. They considered rectangular and exponential profiles for a moving fin. The results obtained were in good agreement with the numerical method. Dogonchi and Ganji [27] used the Differential Transformation method to investigate the temperature distribution in a moving convective-radiative fin with temperature dependent thermal conductivity, heat transfer coefficient and heat generation. Joneidi et al. [28] have used the differential transformation method for studying the effect of temperature-dependent thermal conductivity on the fin efficiency of convective straight fins.

The study of thermal behavior of continuous moving surfaces such as extrusion, hot rolling, glass sheet or wire drawing, casting, powder metallurgy techniques for the fabrication of rod and sheet has become an area of increasing research interests. In the processes such as rolling of strip, extrusion of plastics, drawing of wires and sheets, there is an exchange of heat between material and the surroundings while the material moves through the roller or furnace as shown in Figure 1.

Since, the operations and the thermal configuration of these metal fabrication technologies satisfy the criterion for fin approximation, they can be modeled as continuously moving fins. Consequently, various studies on heat transfer and thermal analysis of a continuously moving fin have been carried out many researchers. Torabi et al. [29] presented the analytical solutions for convective-radiative heat transfer in a continuously moving fin with variable thermal conductivity while Aziz et al. [30] studied the convection-radiation heat transfer from a continuously moving surface with variable thermal conductivity. Aziz and Khani [31] adopted homotopy analysis method to analyze the same problem. 
Singh et al. [32] carried the thermal analysis of a convective-radiative continuously movingin with temper ature-dependent thermal conductivity using wavelet collocation method. Also, Aziz and Torabi [33] analyzed the moving fin with temperature-dependent thermal properties. The effectiveness of spectral element method was demonstrated in the study carried out by Ma et al. [34] on thermal behaviour of longitudinal porous moving fins of different profiles. Furthermore, Singla and Das [35] applied Adomian decomposition method to investigate the thermal analysis in the fin and Bhanja et al. [36] used the same method to study heat transfer enhancement in convective-radiative moving porous moving fins. Also, the heat transfer effects of magnetic on extended surfaces have put forward by Taklifi et al. [37], Hoshyar et al. [38] and Rezazadeh et al. [39]. To the best of the authors' knowledge, the thermal analysis of heat transfer in a moving convective porous fin with temperature-dependent thermal conductivity and internal heat generation using differential transformation and finite difference methods has not been carried out. Therefore, in this present study, a comparative study of thermal analysis of a moving porous convective fin with temperature dependent thermal conductivity and internal heat generation using differential transform and finite difference methods is carried out. Parametric studies were carried out to investigate the effects of thermal geometric and thermo- physical fin parameters on the thermal performance of the porous fin.

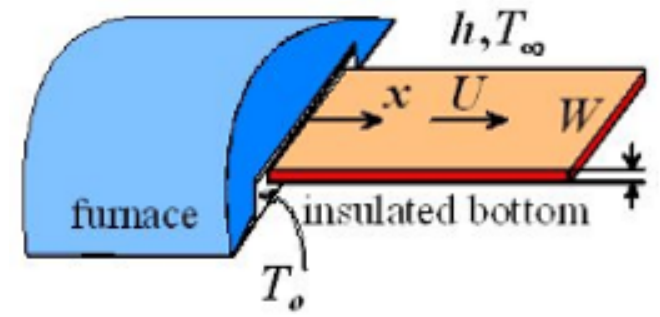

Figure 1. Schematic diagram of rolling and extrusion.

\section{Problem formulation}

Let us consider a straight rectangularfin of constant cros s sectional area, A, with length, b, width W, and thickness, $t^{*}$. The fin is porous to allow the flow of infiltrate through it. The axial coordinate, $\mathrm{x}$ is measured from the base of the fin. The fin is moving horizontally at a constant velocity $U_{X}$.

The following assumptions were made in the development of the model.

* The porous medium is isotropic and homogeneous.

* The porous medium is saturated with single-phase fluid

* The solid matrix and fluid are assumed to be at local thermal equilibrium with each other.

* The interactions between the porous medium and the clear fluid can be simulated by the Darcy formulation.

* The thermal conductivity $K$ and internal heat generation are temperature-dependent and assumed to follow a linear function of temperature $T$. The coefficient of heat transfer of the surrounding medium is assumed to be constant.

* The fin tip is taken as insulated, which means one can neglect the fin tip heat transfer due to its small area.

$$
\frac{d}{d x}\left(k(T) \frac{\partial T}{\partial x}\right)-\frac{\rho C_{p} g k \beta^{*}\left(T-T_{\infty}\right)^{2}}{v t}-\frac{h P\left(T-T_{\infty}\right)}{A}+q^{\prime \prime \prime}(T)=\rho c_{p} V_{w} \frac{d T}{d x}=0
$$

The thermal conductivity and internal heat generation vary linearly with temperature as

$$
\begin{aligned}
& k(T)=k_{0}\left(1+\lambda\left(T-T_{\infty}\right)\right) \\
& q^{*}(T)=q_{o}^{*}\left(1+\gamma\left(T-T_{\infty}\right)\right)
\end{aligned}
$$

Substituting equation (2) and (3) into equation (7), gives

$$
\frac{d}{d x}\left(\left(1+\lambda\left(T-T_{\infty}\right)\right) \frac{d T}{d x}\right)-\frac{\rho C_{p} g k \beta^{*}\left(T-T_{\infty}\right)^{2}}{v k_{o} t}-\frac{h P\left(T-T_{\infty}\right)}{k_{o} A}-\frac{\rho c_{p} u}{k_{o}} \frac{d T}{d x}+\frac{q_{o}^{*}\left(1+\gamma\left(T-T_{\infty}\right)\right)}{k_{o}}=0
$$

Subject to the following boundary conditions;

at

at

$$
\begin{gathered}
x=0, \quad T=T_{b} \\
x=L, \quad \frac{d T}{d x}=0
\end{gathered}
$$


Introducing the following dimensionless variables,

$$
\begin{aligned}
& \theta=\frac{T-T_{s}}{T_{b}-T_{s}} \quad X=\frac{x}{L}, \quad \mathrm{M}^{2}=\frac{h p L^{2}}{k_{o} A} \quad S p=\frac{g k \beta^{*} L^{2}\left(T_{b}-T_{s}\right)}{v \alpha t}=\frac{\operatorname{Dax} R a}{K}\left(\frac{L}{t}\right)^{2} \\
& \alpha=\frac{k_{o}}{\rho C_{p}} P e=\frac{U L}{\alpha} \quad \beta=\lambda\left(T_{b}-T_{\infty}\right) \quad H_{t}=\varepsilon\left(T_{b}-T_{\infty}\right), G=\frac{q_{o}^{*}}{h P\left(T_{b}-T_{\infty}\right)}
\end{aligned}
$$

Where $\mathrm{M}$ is convection parameter that indicates the effect of surface convection of the fin; $S p$ is a porous parameter that indicates the effect of the permeability of the porous medium as well as buoyancy effect, $\beta$ is the dimensionless thermal conductivity and $P e$ is the Peclet number which represent the dimensionless speed of the moving fin $(P e=0$ represents a stationary fin). $G$ represents the heat generation number. $H_{t}$, represents the dimensionless internal heat generation number.

Substituting the dimensionless variables into equation (7), gives the non dimensional governing equation

$$
\frac{d^{2} \theta}{d X^{2}}+\beta \theta \frac{d^{2} \theta}{d X^{2}}+\beta\left(\frac{d \theta}{d X}\right)^{2}-S p \theta^{2}-M^{2} \theta-P e \frac{d \theta}{d X}+M^{2} G\left(1+H_{t} \theta\right)=0
$$

The boundary conditions appear in dimensionless form as

at

$$
X=0, \quad \theta=1
$$

at

$$
X=1, \frac{d \theta}{d X}=0
$$

\section{Method of Solution: Differential Transformation Method}

The governing equation in Eq. (8) is nonlinear and it is difficult to solve exactly. Therefore, in this work, differential transformation method is applied. Considering the efficiency of the method in solving nonlinear differential equation, it is easy to apply the method to the present problem.

Operational properties of differential transformation method.

If $u(t)$ and $v(t)$ are two independent functions with time (t) where $U(p)$ and $V(p)$ are the transformed function corresponding to $u(t)$ and $v(t)$, then it can be proved from the fundamental mathematics operations performed by differential transformation that.

1) If $z(t)=u(t) \pm v(t)$, then $Z(p)=U(p) \pm V(p)$

2) If $z(t)=\alpha u(t)$, then $Z(p)=\alpha U(p)$

3) If $z(t)=\frac{d u(t)}{d t}$, then $Z(p)=(p+1) U(p+1)$

4) If $z(t)=u(t) v(t)$, then $\mathrm{Z}(t)=\sum_{r=0}^{p} V(r) U(p-r)$

5) If $z(t)=u^{m}(t)$, then $\mathrm{Z}(t)=\sum_{r=0}^{p} U^{m-1}(r) U(p-r)$

6) If $z(t)=u(t) v(t)$, then $\mathrm{Z}(k)=\sum_{r=0}^{p}(r+1) V(r+1) U(p-r)$

7) If $z(t)=\frac{d^{2} u(t)}{d t^{2}}$, then $Z(k)=(k+1)(k+2) U(k+2)$

8) If $z(t)=\frac{d^{n} u}{d t^{n}}$, then $Z(k)=(k+1)(k+2) \cdots(k+n) U(k+n)$

9) If $z(t)=\sin (\omega t+\alpha)$, then $Z(k)=\frac{\omega^{k}}{k !} \sin \left(\frac{\pi k}{2 !}+\alpha\right)$

$$
\text { If } z(t)=\cos (\omega t+\alpha) \text {, then } Z(k)=\frac{\omega^{k}}{k !} \cos \left(\frac{\pi k}{2 !}+\alpha\right)
$$


11) If $z(t)=\frac{d^{3} u}{d t^{3}}$, then $Z(k)=(k+1)(k+2)(k+3) U(k+3)$

12) If $z(t)=\frac{d^{4} u}{d t^{4}}$, then $Z(k)=(k+1)(k+2)(k+3)(k+4) U(k+4)$

Using the DTM, we will now attempt using it to solve the governing equation of Eq. 8. Taking the differential transform of Equation (14), we obtain the following recursive relation

$$
\begin{aligned}
& (k+1)(k+2) \theta(k+2)+\beta\left(\sum_{l=0}^{k}(k+2-l)(k+1-l) \theta(l) \theta(k+2-l)\right) \\
& +\beta\left(\sum_{l=0}^{k}(l+1)(k+1-l) \theta(l+1) \theta(k+1-l)\right)-S p\left(\sum_{l=0}^{k} \theta(l) \theta(k-l)\right) \\
& -M^{2} \theta(k)-P e(k+1) \theta(k+1) \\
& +M^{2} G \delta(k)+M^{2} G H_{t} \theta(k)=0
\end{aligned}
$$

Where $\theta(k)$ is the differential transform of $\theta(x)$. Taking the dimensional differential transform of the boundary conditions in Eqs. (9) and (10) we obtain the following transformations respectively,

$$
\theta(0)=1 ; \theta(1)=a
$$

the constant ' $a$ ' can be determined from the boundary (9) after obtaining the series solution. Analysis Eq. (11) and substituting Eq. (12) into Eq. (11), we obtain the following

$$
\begin{aligned}
& \theta[1]=a ; \theta[0]=1 \text {; } \\
& \theta[2]=\frac{M^{2}-G M^{2}+a P e-a^{2} \beta-G M^{2} H_{t}+S_{p}}{2(1+\beta)} ; \\
& a M^{2}+M^{2} P e-G M^{2} P e+a P e^{2}-2 a M^{2} \beta+3 a G M^{2} \beta \\
& \theta[3]=\frac{-4 a^{2} P e \beta+3 a^{3} \beta^{2}-a G M^{2} H_{t}-G M^{2} P e H_{t}+2 a G M^{2} \beta H_{t}+2 a S_{p}+P e S_{p}-a \beta S_{p}}{6(1+\beta)^{2}} ; \\
& \left.\theta[4]=\left[\begin{array}{l}
\frac{M^{2}\left(M^{2}-G M^{2}+a P e-a^{2} \beta-G M^{2} H_{t}+S_{p}\right)}{2(1+\beta)} \\
-\frac{G M^{2} H_{t}\left(M^{2}-G M^{2}+a P e-a^{2} \beta-G M^{2} H_{t}+S_{p}\right)}{2(1+\beta)}- \\
\frac{\beta\left(M^{2}-G M^{2}+a P e-a^{2} \beta-G M^{2} H_{t}+S_{p}\right)^{2}}{2(1+\beta)^{2}} \\
\begin{array}{l}
P e\left(\begin{array}{l}
a M^{2}+M^{2} P e-G M^{2} P e+a P e^{2}-2 a M^{2} \beta+3 a G M^{2} \beta-4 a^{2} P e \beta \\
+3 a^{3} \beta^{2}-a G M^{2} H_{t}-G M^{2} P e H_{t}+2 a G M^{2} \beta H_{t}+2 a S_{p}+P e S_{p}-a \beta S_{p}
\end{array}\right) \\
2(1+\beta)^{2}
\end{array} \\
\begin{array}{l}
a \beta\left(\begin{array}{l}
a M^{2}+M^{2} P e-G M^{2} P e+a P e^{2}-2 a M^{2} \beta \\
+3 a G M^{2} \beta-4 a^{2} P e \beta+3 a^{3} \beta^{2}-a G M^{2} H_{t} \\
-G M^{2} P e H_{t}+2 a G M^{2} \beta H_{t}+2 a S_{p}+P e S_{p}-a \beta S_{p}
\end{array}\right) \\
(1+\beta)^{2}
\end{array} \\
S_{p}\left(\begin{array}{l}
\left.a^{2}+\frac{M^{2}-G M^{2}+a P e-a^{2} \beta-G M^{2} H_{t}+S_{p}}{1+\beta}\right) \\
{\left[\left(\begin{array}{l}
\left(M^{2}-G M^{2}+a P e-a^{2} \beta-G M^{2} H_{t}+S_{p}\right)^{2} \\
(1+\beta)^{2}
\end{array}\right)+\right.} \\
-\beta\left[\begin{array}{l}
a M^{2}+M^{2} P e-G M^{2} P e+a P e^{2}-2 a M^{2} \beta \\
a\left(3 a G M^{2} \beta-4 a^{2} P e \beta+3 a^{3} \beta^{2}-a G M^{2} H_{t}\right. \\
-G M^{2} P e H_{t}+2 a G M^{2} \beta H_{t}+2 a S_{p}+P e S_{p}-a \beta S_{p}
\end{array}\right) \\
(1+\beta)^{2}
\end{array}\right.
\end{array}\right]\right)
\end{aligned}
$$


From the definition and basic principle of DTM, we obtain the infinite series solution given by

$$
\begin{aligned}
& \theta(X)=\theta(0)+\theta(1) X+\theta(2) X^{2}+\theta(3) X^{3}+\theta(4) X^{4}+\theta(5) X^{5}+\ldots . . \\
& \theta(X)=1+a X+\left(\frac{M^{2}-G M^{2}+a P e-a^{2} \beta-G M^{2} H_{t}+S_{p}}{2(1+\beta)}\right) X^{2} \\
& +\frac{\left(\begin{array}{l}
a M^{2}+M^{2} P e-G M^{2} P e+a P e^{2}-2 a M^{2} \beta+3 a G M^{2} \beta \\
-4 a^{2} P e \beta+3 a^{3} \beta^{2}-a G M^{2} H_{t}-G M^{2} P e H_{t}+2 a G M^{2} \beta H_{t} \\
+2 a S_{p}+P e S_{p}-a \beta S_{p}
\end{array}\right)}{6(1+\beta)^{2}} X^{3} \\
& \int \frac{M^{2}\left(M^{2}-G M^{2}+a P e-a^{2} \beta-G M^{2} H_{t}+S_{p}\right)}{2(1+\beta)} \\
& -\frac{G M^{2} H_{t}\left(M^{2}-G M^{2}+a P e-a^{2} \beta-G M^{2} H_{t}+S_{p}\right)}{2(1+\beta)}- \\
& \frac{\beta\left(M^{2}-G M^{2}+a P e-a^{2} \beta-G M^{2} H_{t}+S_{p}\right)^{2}}{2(1+\beta)^{2}} \\
& +\frac{P e\left(\begin{array}{l}
a M^{2}+M^{2} P e-G M^{2} P e+a P e^{2}-2 a M^{2} \beta+3 a G M^{2} \beta-4 a^{2} P e \beta \\
+3 a^{3} \beta^{2}-a G M^{2} H_{t}-G M^{2} P e H_{t}+2 a G M^{2} \beta H_{t}+2 a S_{p}+P e S_{p}-a \beta S_{p}
\end{array}\right)}{2(1+\beta)^{2}}- \\
& +\frac{1}{12+12 \beta} \mid \begin{array}{c}
\frac{a \beta\left(\begin{array}{l}
a M^{2}+M^{2} P e-G M^{2} P e+a P e^{2}-2 a M^{2} \beta \\
+3 a G M^{2} \beta-4 a^{2} P e \beta+3 a^{3} \beta^{2}-a G M^{2} H_{t} \\
-G M^{2} P e H_{t}+2 a G M^{2} \beta H_{t}+2 a S_{p}+P e S_{p}-a
\end{array}\right.}{(1+\beta)^{2}} \\
S_{p}\left(a^{2}+\frac{M^{2}-G M^{2}+a P e-a^{2} \beta-G M^{2} H_{t}+S_{p}}{1+\beta}\right)
\end{array} \\
& {\left[\left(\frac{\left(M^{2}-G M^{2}+a P e-a^{2} \beta-G M^{2} H_{t}+S_{p}\right)^{2}}{(1+\beta)^{2}}\right)+\right.} \\
& -\beta\left(\begin{array}{l}
a M^{2}+M^{2} P e-G M^{2} P e+a P e^{2}-2 a M^{2} \beta \\
a+3 a G M^{2} \beta-4 a^{2} P e \beta+3 a^{3} \beta^{2}-a G M^{2} H_{t}
\end{array}\right. \\
& -G M^{2} P e H_{t}+2 a G M^{2} \beta H_{t}+2 a S_{p}+P e S_{p}-a \beta S_{p} \\
& (1+\beta)^{2}
\end{aligned}
$$

\section{Method of Solution using Finite Difference Method}

Consider first a mesh in space formed by points separated by constant spacing $\Delta \mathrm{x}$ as shown in Figure 2 . The mesh points in space are are $x_{1}, x_{2}, x_{3} \ldots x_{N-1}, x_{N}$. Note that nodes $x_{1}$ and $x_{N}$ are boundary nodes while all other nodes are interior nodes. 


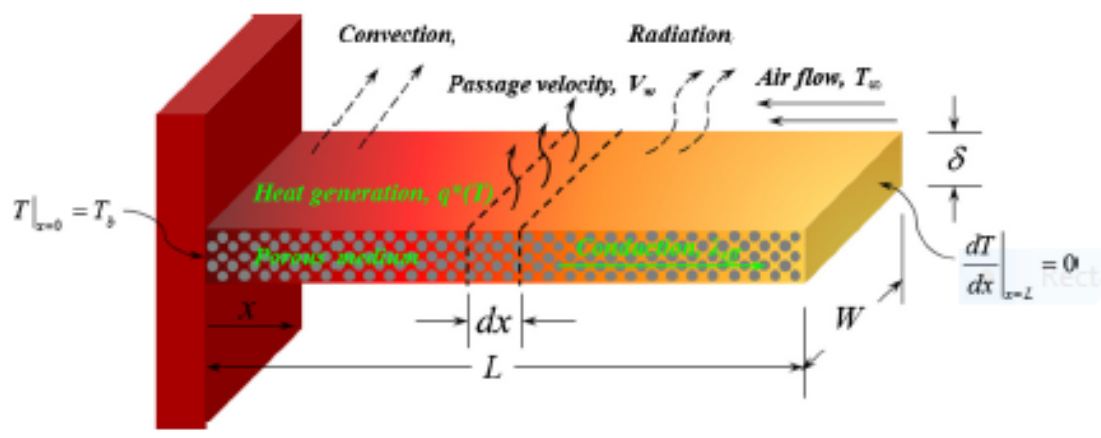

Figure 2. Schematic of the convective longitudinal porous fin [34].

The procedures are shown below

$$
\begin{aligned}
& \frac{\theta_{i+1}-2 \theta_{i}+\theta_{i-1}}{\Delta^{2} x}+\beta \theta_{i}\left(\frac{\theta_{i+1}-2 \theta_{i}+\theta_{i-1}}{\Delta^{2} x}\right)+\beta\left(\frac{\theta_{i+1}-\theta_{i-1}}{2 \Delta x}\right)^{2} \\
& -S_{p} \theta_{i}^{2}-M^{2} \theta_{i}-P e\left(\frac{\theta_{i+1}-\theta_{i-1}}{2 \Delta x}\right)+M^{2} G\left(1+H_{t} \theta_{i}\right)=0
\end{aligned}
$$

After applying the finite difference approximation and grouping and rearranging the terms, the final algebraic form of the finite difference equation becomes

$$
\begin{aligned}
& \theta_{i+1}-2 \theta_{i}+\theta_{i-1}+\beta \theta_{i}\left(\theta_{i+1}-2 \theta_{i}+\theta_{i-1}\right)+\frac{\beta}{4} \theta_{i+1}^{2}-\frac{\beta}{2} \theta_{i+1} \theta_{i-1}+\frac{\beta}{4} \theta_{i-1}^{2}-S_{p} \theta_{i}^{2}\left(\Delta^{2} x\right) \\
& -M^{2} \theta_{i}\left(\Delta^{2} x\right)-P e\left(\frac{\theta_{i+1}-\theta_{i-1}}{2 \Delta x}\right)\left(\Delta^{2} x\right)+M^{2} G\left(1+H_{t} \theta_{i}\right)\left(\Delta^{2} x\right)=0
\end{aligned}
$$

It could be seen that the central difference approximation is used for the differentials, so Equ. (15) is only valid for interior nodes $(i=2: N$ - 1 ) because the central difference approximation cannot be applied at the end points. Thus, for the 48 interior nodes (the remaining 2 nodes of the 50 nodes used in this work are at the boundaries of the fin), we have for

$$
\begin{aligned}
& \theta_{3}-2 \theta_{2}+\theta_{1}+\beta \theta_{2}\left(\theta_{3}-2 \theta_{2}+\theta_{1}\right)+\frac{\beta}{4} \theta_{3}^{2}-\frac{\beta}{2} \theta_{3} \theta_{1}+\frac{\beta}{4} \theta_{1}^{2}-S_{p} \theta_{2}^{2}\left(\Delta^{2} x\right) \\
& i=2 \\
& -M^{2} \theta_{2}\left(\Delta^{2} x\right)-\frac{P e(\Delta x)}{2} \theta_{3}+\frac{P e(\Delta x)}{2} \theta_{1}+M^{2} G\left(1+H_{t} \theta_{2}\right)\left(\Delta^{2} x\right)=0 \\
& i=3 \\
& \theta_{4}-2 \theta_{3}+\theta_{2}+\beta \theta_{3}\left(\theta_{4}-2 \theta_{3}+\theta_{2}\right)+\frac{\beta}{4} \theta_{4}^{2}-\frac{\beta}{2} \theta_{4} \theta_{2}+\frac{\beta}{4} \theta_{2}^{2}-S_{p} \theta_{3}^{2}\left(\Delta^{2} x\right) \\
& -M^{2} \theta_{3}\left(\Delta^{2} x\right)-\frac{P e(\Delta x)}{2} \theta_{4}+\frac{P e(\Delta x)}{2} \theta_{2}+M^{2} G\left(1+H_{t} \theta_{3}\right)\left(\Delta^{2} x\right)=0 \\
& \theta_{5}-2 \theta_{4}+\theta_{3}+\beta \theta_{4}\left(\theta_{5}-2 \theta_{4}+\theta_{3}\right)+\frac{\beta}{4} \theta_{5}^{2}-\frac{\beta}{2} \theta_{5} \theta_{3}+\frac{\beta}{4} \theta_{3}^{2}-S_{p} \theta_{4}^{2}\left(\Delta^{2} x\right) \\
& i=4 \\
& -M^{2} \theta_{4}\left(\Delta^{2} x\right)-\frac{P e(\Delta x)}{2} \theta_{5}+\frac{P e(\Delta x)}{2} \theta_{3}+M^{2} G\left(1+H_{t} \theta_{4}\right)\left(\Delta^{2} x\right)=0 \\
& i=N-2 ; \quad \theta_{N-1}-2 \theta_{N-2}+\theta_{N-3}+\beta \theta_{N-2}\left(\theta_{N-1}-2 \theta_{N-2}+\theta_{N-3}\right)+\frac{\beta}{4} \theta_{N-1}^{2}-\frac{\beta}{2} \theta_{N-1} \theta_{N-3}+\frac{\beta}{4} \theta_{N-3}^{2}-S_{p} \theta_{N-2}^{2}\left(\Delta^{2} x\right) \\
& -M^{2} \theta_{N-2}\left(\Delta^{2} x\right)-\frac{P e(\Delta x)}{2} \theta_{N-1}+\frac{P e(\Delta x)}{2} \theta_{N-3}+M^{2} G\left(1+H_{t} \theta_{N-2}\right)\left(\Delta^{2} x\right)=0 \\
& i=N-1 \quad \theta_{N}-2 \theta_{N-1}+\theta_{N-2}+\beta \theta_{N-1}\left(\theta_{N}-2 \theta_{N-1}+\theta_{N-2}\right)+\frac{\beta}{4} \theta_{N}^{2}-\frac{\beta}{2} \theta_{N} \theta_{N-2}+\frac{\beta}{4} \theta_{N-2}^{2}-S_{p} \theta_{N-1}^{2}\left(\Delta^{2} x\right) \\
& -M^{2} \theta_{N-1}\left(\Delta^{2} x\right)-\frac{P e(\Delta x)}{2} \theta_{N}+\frac{P e(\Delta x)}{2} \theta_{N-2}+M^{2} G\left(1+H_{t} \theta_{N-1}\right)\left(\Delta^{2} x\right)=0
\end{aligned}
$$

For the first node, $i=1$, equation (15) can be used directly noting that $\theta_{i-1}=\theta_{0}=1$, the fin's base temperature. 


$$
\begin{array}{ll}
i=1 \quad & \theta_{2}-2 \theta_{1}+\theta_{0}+\beta \theta_{1}\left(\theta_{2}-2 \theta_{1}+\theta_{0}\right)+\frac{\beta}{4} \theta_{2}^{2}-\frac{\beta}{2} \theta_{2} \theta_{0}+\frac{\beta}{4} \theta_{0}^{2}-S_{p} \theta_{1}^{2}\left(\Delta^{2} x\right) \\
& -M^{2} \theta_{1}\left(\Delta^{2} x\right)-\frac{P e(\Delta x)}{2} \theta_{2}+\frac{P e(\Delta x)}{2} \theta_{0}+M^{2} G\left(1+H_{t} \theta_{1}\right)\left(\Delta^{2} x\right)=0
\end{array}
$$

However, the last node at the tip of the fin is treated as a special case i.e. for $i=N, \theta_{N+1}$ is not defined. Although, this situation can be handled in several ways, possibly the easiest way is to generate a backward approximation to the desired derivatives at $X=1$. After algebraic manipulations, we arrived at

$$
\begin{aligned}
& \frac{\theta_{N-2}-\theta_{N}}{2 \Delta^{2} x}+\beta \theta_{N}\left(\frac{\theta_{N-2}-\theta_{N}}{2 \Delta^{2} x}\right)+\beta\left(\frac{\theta_{N}-\theta_{N-1}}{2 \Delta x}\right)^{2}-S_{p} \theta_{N}^{2}\left(\Delta^{2} x\right) \\
& -M^{2} \theta_{N}\left(\Delta^{2} x\right)-P e\left(\frac{\theta_{N}-\theta_{N-1}}{2 \Delta x}\right)+M^{2} G\left(1+H_{t} \theta_{N}\right)\left(\Delta^{2} x\right)=0
\end{aligned}
$$

Which could be simplified as

$$
\begin{aligned}
& \theta_{N-2}-\theta_{N}+\beta \theta_{N}\left(\theta_{N-2}-\theta_{N}\right)+\frac{\beta}{2}\left(\theta_{N}-\theta_{N-1}\right)^{2}-2 S_{p} \theta_{N}^{2}\left(\Delta^{2} x\right) \\
& -2 M^{2} \theta_{N}\left(\Delta^{2} x\right)-2 P e\left(\frac{\theta_{N}-\theta_{N-1}}{2 \Delta x}\right)\left(\Delta^{2} x\right)+2 M^{2} G\left(1+H_{t} \theta_{N}\right)\left(\Delta^{2} x\right)=0
\end{aligned}
$$

The FDM results in a set of 50 non-linear algebraic equations (since 50 nodes are chosen to be used in this work) from Equs. (16), Equ. (17) and Equ. (19) These equations are required are solved simultaneously. The systems of the non-linear equations are solved with the aid of MATLAB using fsolve.

If the nonlinear terms are removed, the dimensionless form of the governing equation reduces to

$$
\frac{\partial^{2} \theta}{\partial X^{2}}-P e \frac{\partial \theta}{\partial X}-M^{2}\left(1-G H_{t}\right) \theta=-M^{2} G
$$

And the exact analytical solution is given by

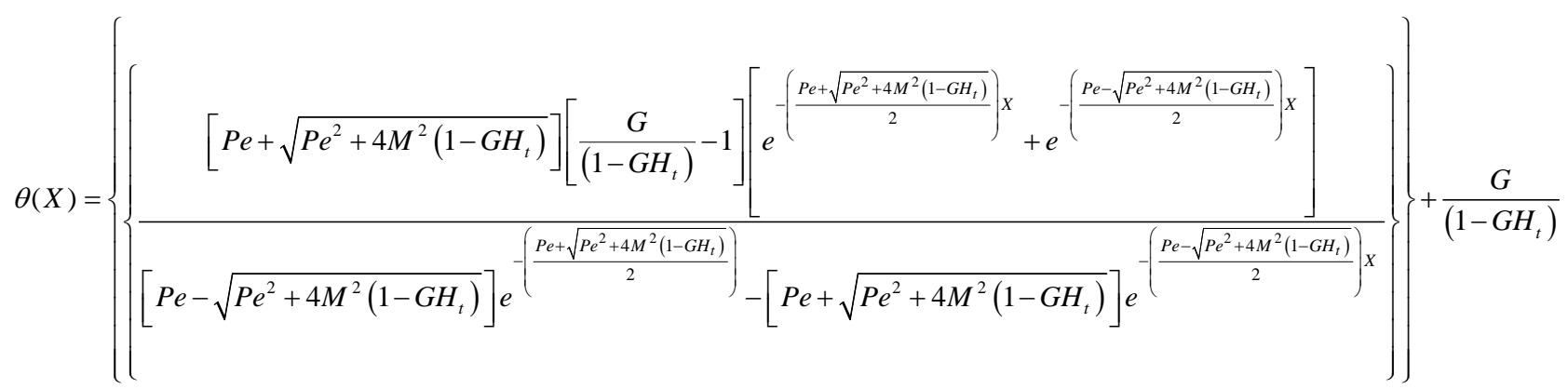

\section{Parameters of Engineering Interests}

The base heat flow is given by

$$
q_{b}=\left.k A \frac{\partial T}{\partial X}\right|_{x=0}
$$

Which may be expressed in dimensionless form as

The total heat flux of the fin is given by:

$$
Q_{b}=\frac{q_{b} L}{k_{0} A\left(T_{b}-T_{s}\right)}=\left.(1+\beta \theta) \frac{\partial \theta}{\partial X}\right|_{X=0}
$$

$$
q_{T}=(1+\beta \theta) \frac{\partial \theta}{\partial X}
$$

The fin efficiency may be defined as the convective heat loss divided by the convective heat loss if the entire fin was at the temperature of its base, i.e. 


$$
\begin{gathered}
\eta=\frac{Q_{f}}{Q_{\max }} \\
Q_{f}=\int_{0}^{L}\left(\frac{\rho C_{p} g k A \beta^{*}\left(T-T_{\infty}\right)^{2}}{v t}+h P\left(T-T_{\infty}\right)+\rho c_{p} u A \frac{d T}{d x}\right) d X \\
Q_{\max }=\frac{\rho C_{p} g k A \beta^{*}\left(T_{b}-T_{\infty}\right)^{2} L}{v t}+h P L\left(T_{b}-T_{\infty}\right)+\left.\rho c_{p} u A L \frac{d T}{d x}\right|_{x=0} \\
\eta=\frac{Q_{f}}{Q_{\max }}=\frac{\int_{0}^{L}\left(\frac{\rho C_{p} g k A \beta^{*}\left(T-T_{\infty}\right)^{2}}{v t}+h P\left(T-T_{\infty}\right)+\rho c_{p} u A \frac{d T}{d x}\right) d X}{\frac{\rho C_{p} g k A \beta^{*}\left(T_{b}-T_{\infty}\right)^{2} L}{v t}+h P L\left(T_{b}-T_{\infty}\right)+\left.\rho c_{p} u A L \frac{d T}{d x}\right|_{x=0}}
\end{gathered}
$$

Which may be expressed in dimensionless form as

$$
\eta=\frac{\int_{0}^{1}\left[S p \theta^{2}+M^{2} \theta+P e \frac{d \theta}{d X}\right] d X}{S p+M^{2}+\left.P e \frac{d \theta}{d X}\right|_{X=0}}
$$

\section{Results and Discussion}

The developed analytical models are simulated in MATHEMATICA and the results are given in Figs. 3-15. These results show the parametric studies of the effects of the thermogeometric parameter $M$, the thermal conductivity $\beta$, the porosity factor $S_{p}$, the Peclet number $P e$, the internal heat generation $H t$, and heat generation parameter $G$ on the temperature distribution, rate of heat transfer and efficiency of the porous fin. However, prior to this parametric study, it is

\begin{tabular}{|c|c|c|c|c|c|c|}
\hline \multicolumn{7}{|c|}{$\begin{array}{l}\text { The results of DTM and Numerical methods for } \theta(X) \text { for } S p=0.1 ; \beta=0.2 ; P e=0.5 ; H t=0.1, G=0.1 \text {; } \\
\qquad \theta(X)\end{array}$} \\
\hline \multirow{2}{*}{$X$} & \multicolumn{3}{|c|}{$\mathrm{M}=0.1$} & \multicolumn{3}{|c|}{$\mathrm{M}=0.2$} \\
\hline & $F D M$ & DTM & $|F D M-D T M|$ & $F D M$ & DTM & $|F D M-D T M|$ \\
\hline 0.00 & 1.00000000 & 1.00000000 & 0.00000000 & 1.00000000 & 1.00000000 & 0.00000000 \\
\hline 0.05 & 0.99653123 & 0.99653118 & 0.00000005 & 0.99570830 & 0.99570820 & 0.00000009 \\
\hline 0.10 & 0.99321531 & 0.99321522 & 0.00000009 & 0.99160672 & 0.99160654 & 0.00000018 \\
\hline 0.15 & 0.99005425 & 0.99005413 & 0.00000012 & 0.98769759 & 0.98769735 & 0.00000024 \\
\hline 0.20 & 0.98705019 & 0.98705005 & 0.00000014 & 0.98398341 & 0.98398313 & 0.00000028 \\
\hline 0.25 & 0.98420537 & 0.98420521 & 0.00000015 & 0.98046681 & 0.98046649 & 0.00000032 \\
\hline 0.30 & 0.98152213 & 0.98152196 & 0.00000017 & 0.97715056 & 0.97715021 & 0.00000035 \\
\hline 0.35 & 0.97900293 & 0.97900276 & 0.00000017 & 0.97403758 & 0.97403722 & 0.00000036 \\
\hline 0.40 & 0.97665037 & 0.97665020 & 0.00000017 & 0.97113096 & 0.97113059 & 0.00000037 \\
\hline 0.45 & 0.97446713 & 0.97446696 & 0.00000017 & 0.96843393 & 0.96843356 & 0.00000037 \\
\hline 0.50 & 0.97245605 & 0.97245588 & 0.00000017 & 0.96594988 & 0.96594950 & 0.00000038 \\
\hline 0.55 & 0.97062007 & 0.97061990 & 0.00000017 & 0.96368236 & 0.96368199 & 0.00000037 \\
\hline 0.60 & 0.96896226 & 0.96896209 & 0.00000017 & 0.96163511 & 0.96163473 & 0.00000038 \\
\hline 0.65 & 0.96748584 & 0.96748567 & 0.00000017 & 0.95981200 & 0.95981162 & 0.00000038 \\
\hline 0.70 & 0.96619414 & 0.96619397 & 0.00000017 & 0.95821711 & 0.95821672 & 0.00000039 \\
\hline 0.75 & 0.96509065 & 0.96509046 & 0.00000019 & 0.95685467 & 0.95685427 & 0.00000040 \\
\hline 0.80 & 0.96417897 & 0.96417877 & 0.00000020 & 0.95572911 & 0.95572870 & 0.00000041 \\
\hline 0.85 & 0.96346287 & 0.96346265 & 0.00000022 & 0.95484505 & 0.95484461 & 0.00000044 \\
\hline 0.90 & 0.96294626 & 0.96294600 & 0.00000026 & 0.95420727 & 0.95420680 & 0.00000047 \\
\hline 0.95 & 0.96263318 & 0.96263288 & 0.00000030 & 0.95382078 & 0.95382027 & 0.00000051 \\
\hline 1.00 & 0.96252787 & 0.96252750 & 0.00000037 & 0.95369076 & 0.95369019 & 0.00000057 \\
\hline
\end{tabular}
required to verify the developed analytical solutions. Hence, a numeric scheme based on the finite difference method has been used for the validation purpose. Figure is drawn for the validation of the present analytical method. Figure shows that both analytical method and numerical method are in good agreement with each other.

Table 1. Comparison of results 


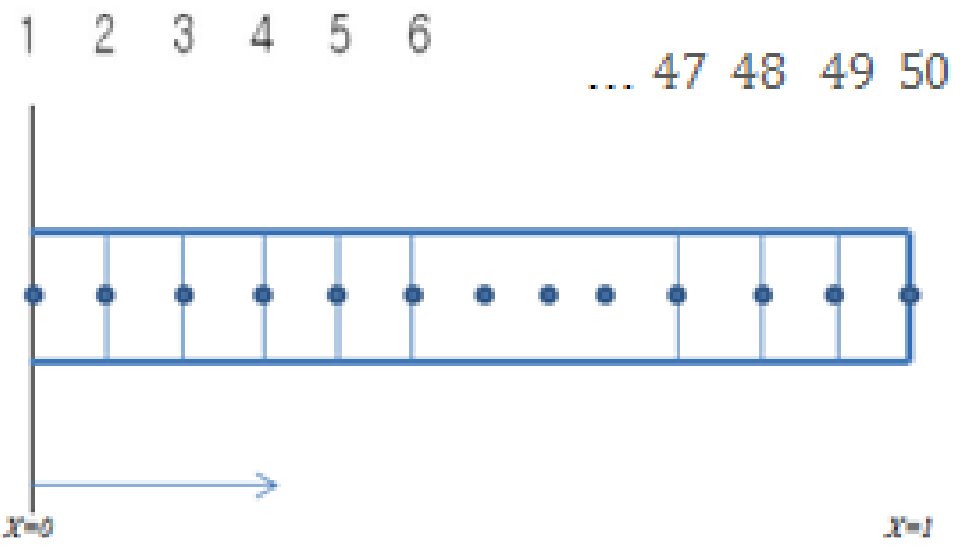

Figure 3. Nodal representation for finite difference method.

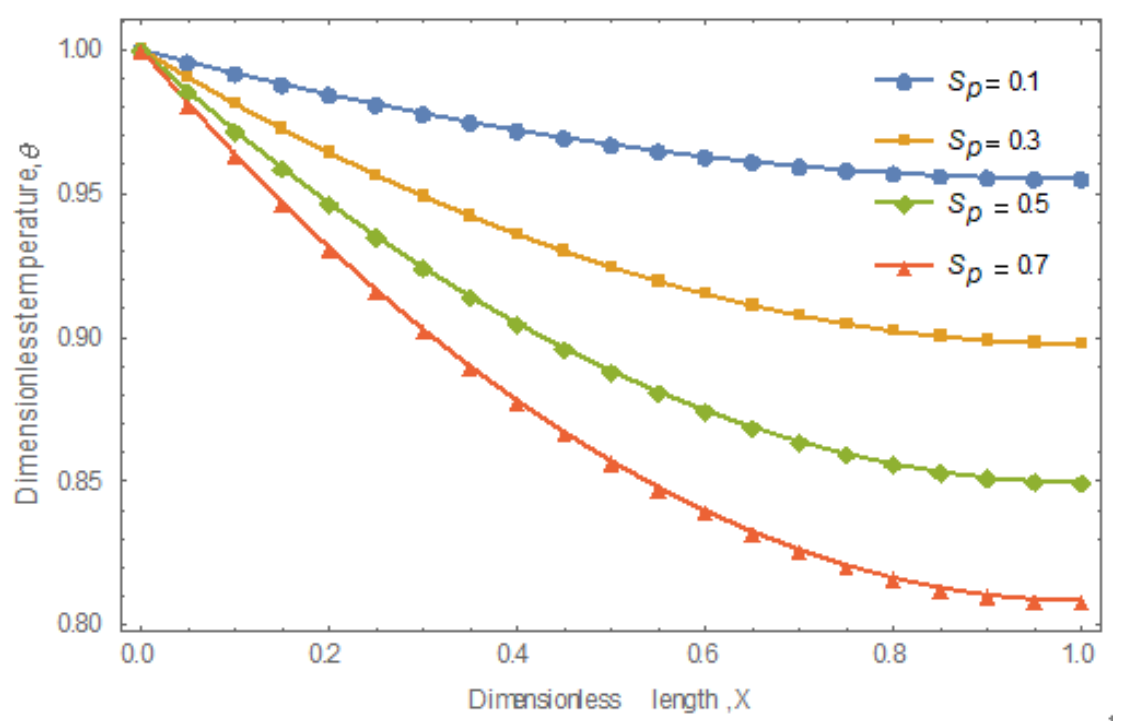

Figure 4. Effect of porosity on temperature distribution in the fin parameters when $\beta=0.2 ; M=0.3 ; P e=0.5 ; G=$ $0.4 ; H_{t}=0.6$.

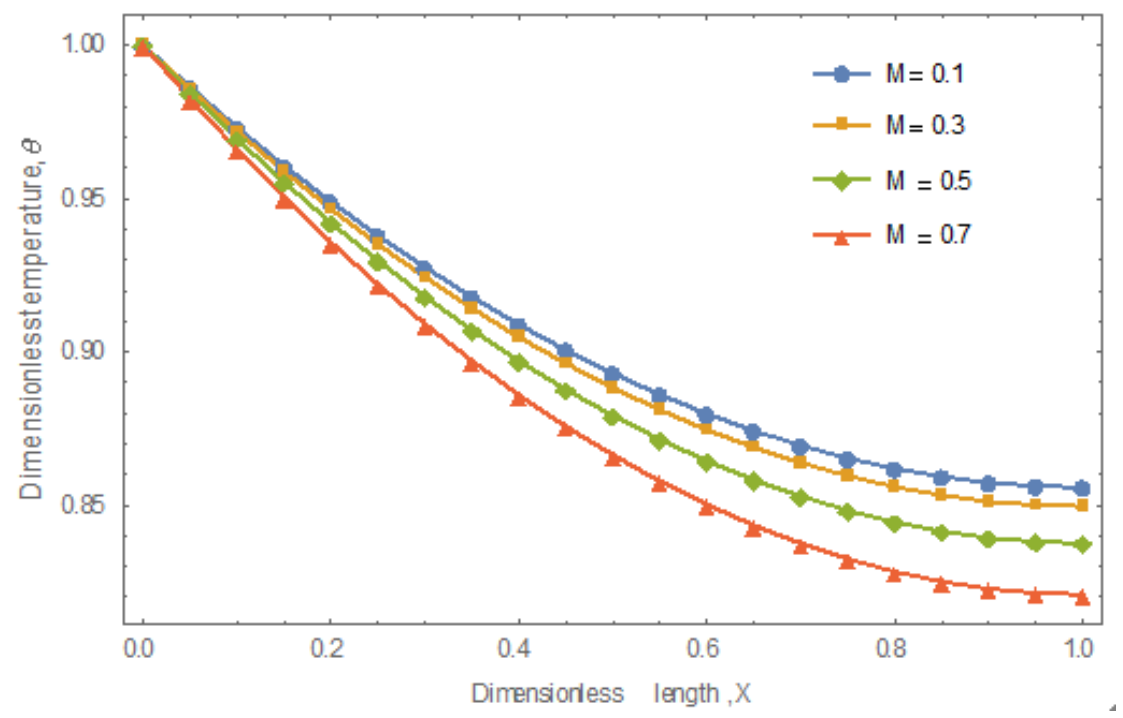

Figure 5. Effect of conductive-convective parameter on temperature distribution in the fin when $\beta=0.2 ; S_{p}=0.5 ;$ Pe $=$ $0.5 ; G=0.4 ; H_{t}=0.6$. 


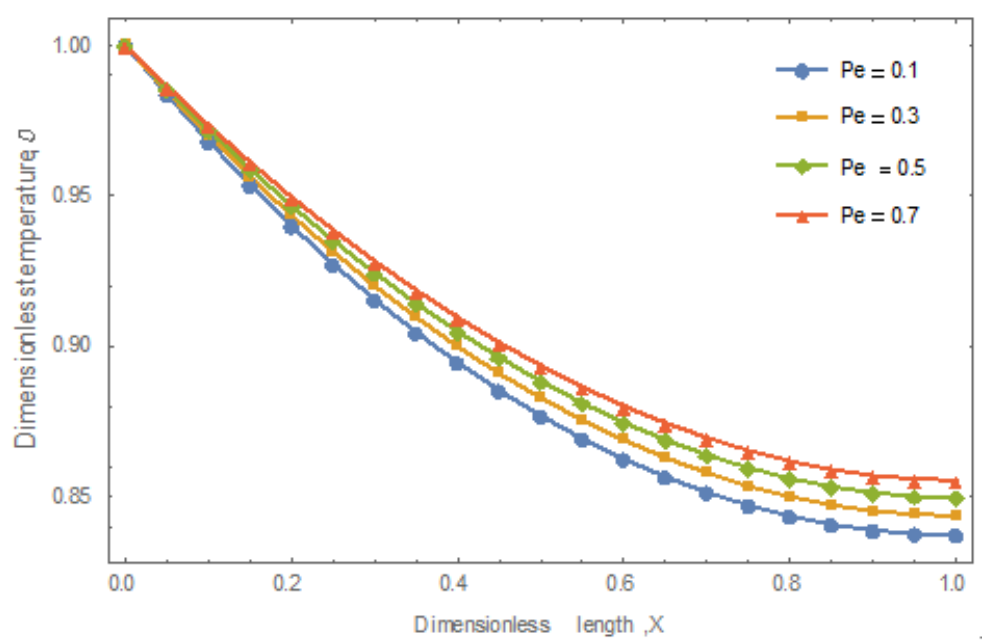

Figure 6. Effect of Peclet number on temperature distribution in the fin when $\beta=0.2 ; M=0.3 ; S_{p}=0.5 ; G=0.4 ; H_{t}=$ 0.6 .

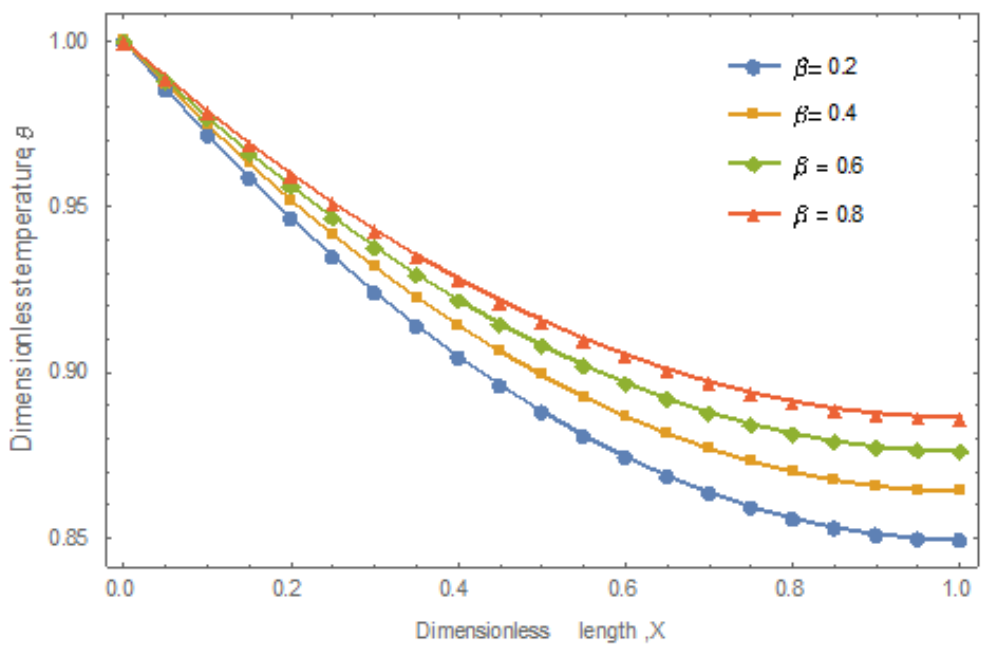

Figure 7. Effect of nonlinear thermal conductivity parameter on the temperature distribution in the fin when $S_{p}=0.5 ; \mathrm{Pe}=$ $0.5 ; G=0.4 ; H_{t}=0.6 ; M=0.3$.

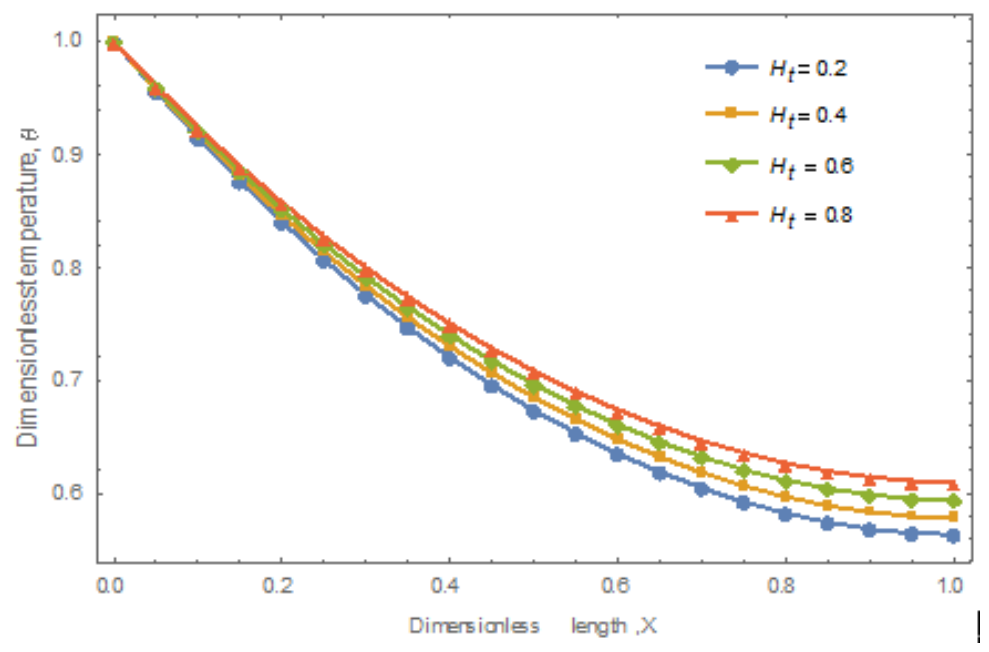

Figure 8. Effect of temperature-dependent internal heat parameter on the temperature distribution in the fin when $\beta=2 ; M=2 ; S_{p}=5 ; \mathrm{Pe}=2 ; G=0.4$. 


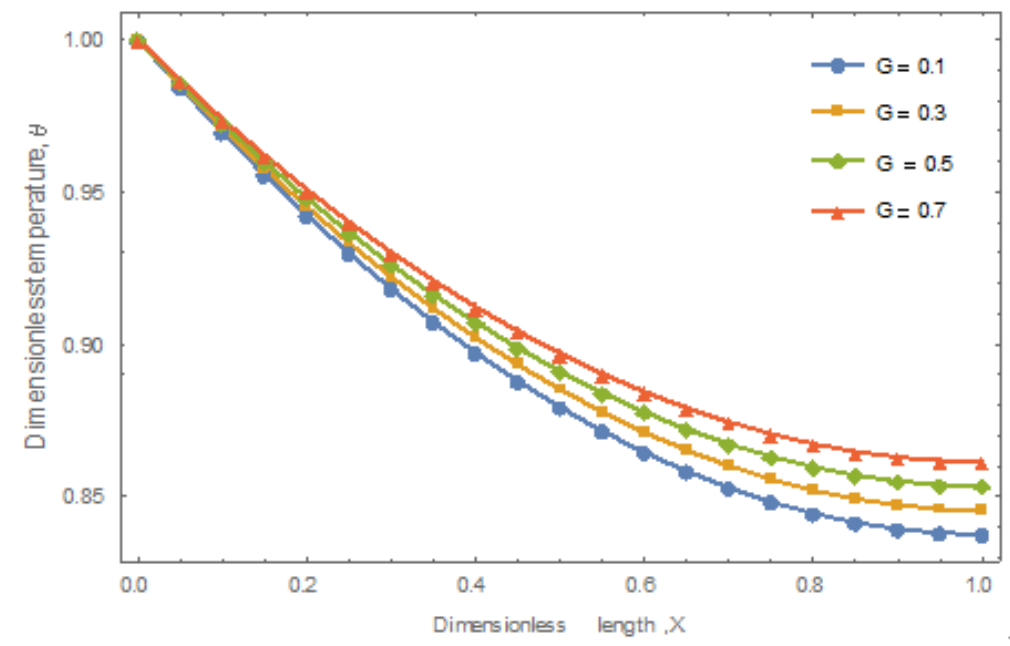

Figure 9. Effect of temperature-dependent internal heat parameter on the temperature distribution in the fin when $\beta=0.2 ; M=0.3 ; S_{p}=0.5 ; \mathrm{Pe}=0.5 ; H_{t}=0.6$.

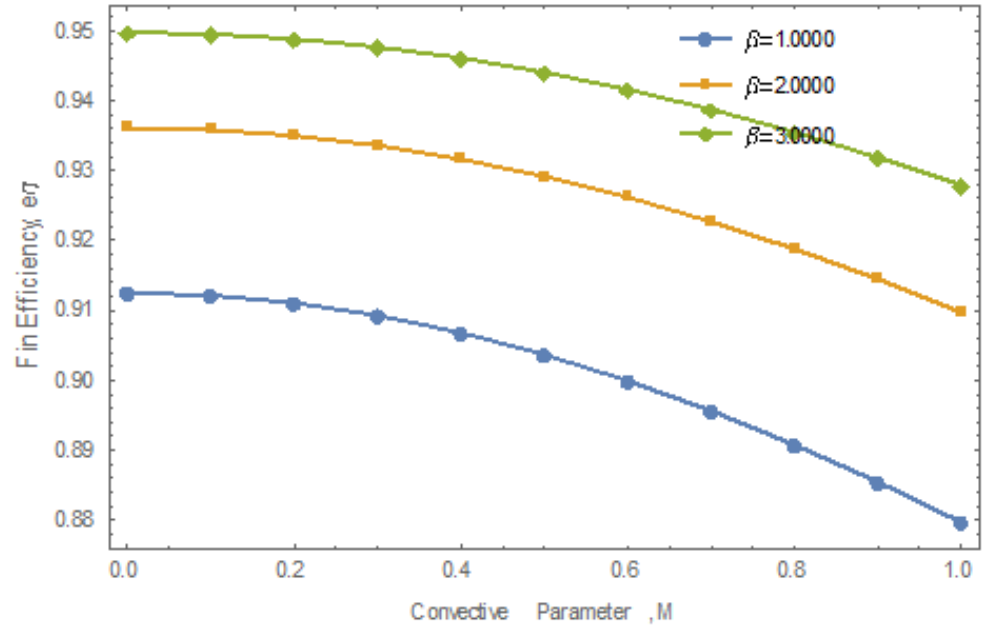

Figure 10. Effect of conductive-convective parameter and nonlinear thermal conductivity on the thermal efficiency when $H_{t}=0.6 ; S_{p}=0.7 ; G=0.4 ; \mathrm{Pe}=0.5$.

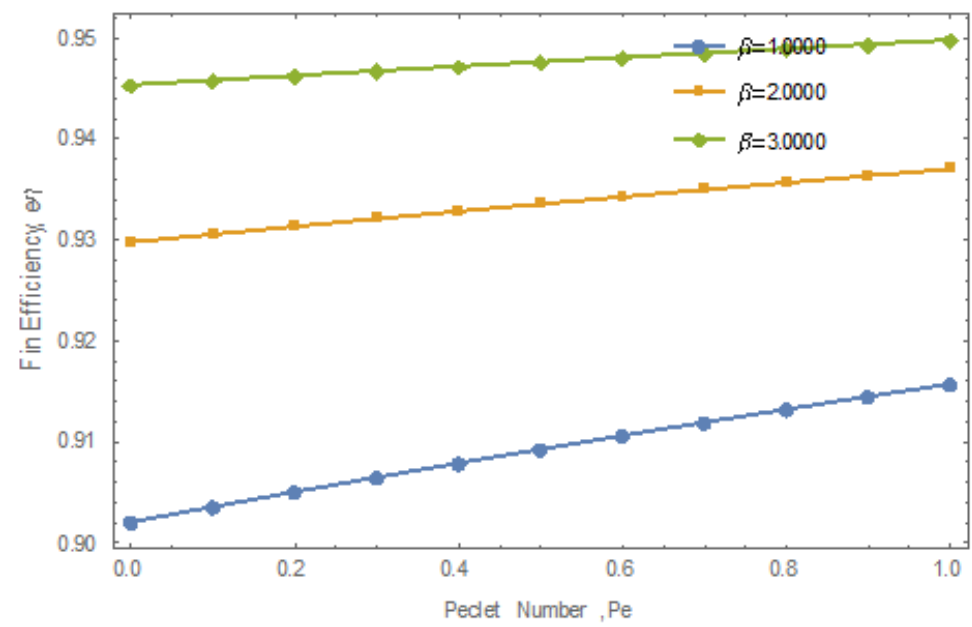

Figure 11. Effect of nonlinear thermal conductivity parameter and Peclet number on the thermal efficiency when $H_{t}=$ $0.6 ; M=0.3 ; S_{p}=0.7 ; G=0.4$. 


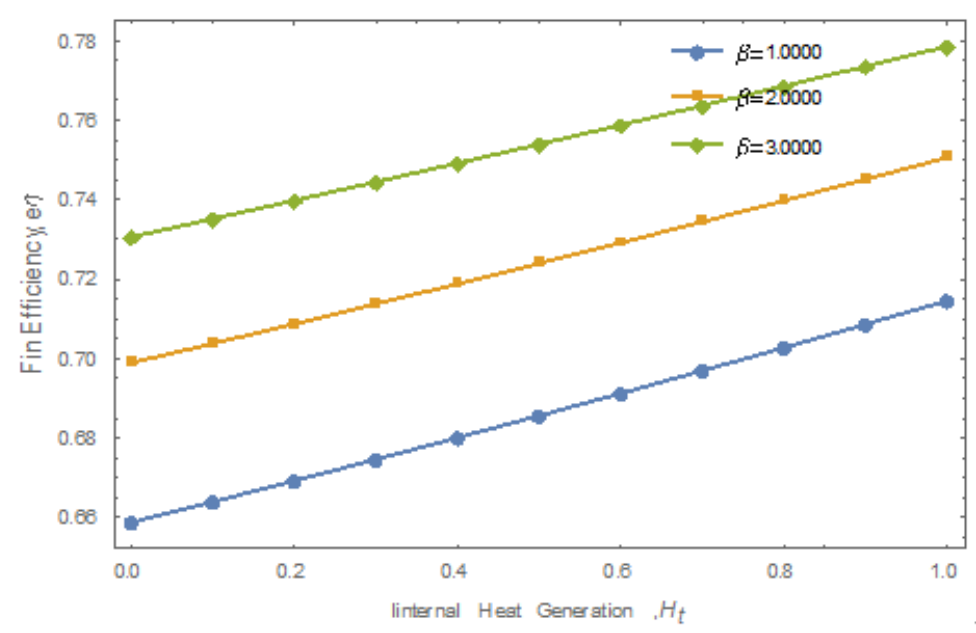

Figure 12. Effect of nonlinear thermal conductivity and internal heat generation parameter on the thermal efficiency when $M=2 ; S_{p}=5 ; G=0.4 ; \mathrm{Pe}=2$.

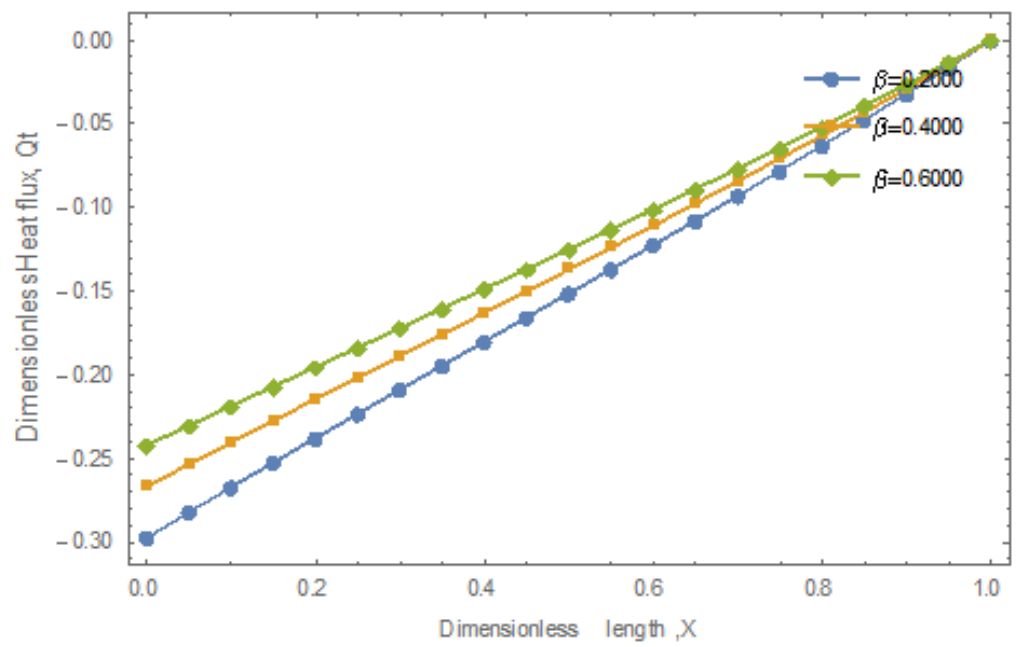

Figure 13. Effect of nonlinear thermal conductivity on the heat flux across fin length when $H_{t}=0.6 ; M=0.3 ; S_{p}=0.5 ; G=$ $0.4 ; \mathrm{Pe}=0.5$;

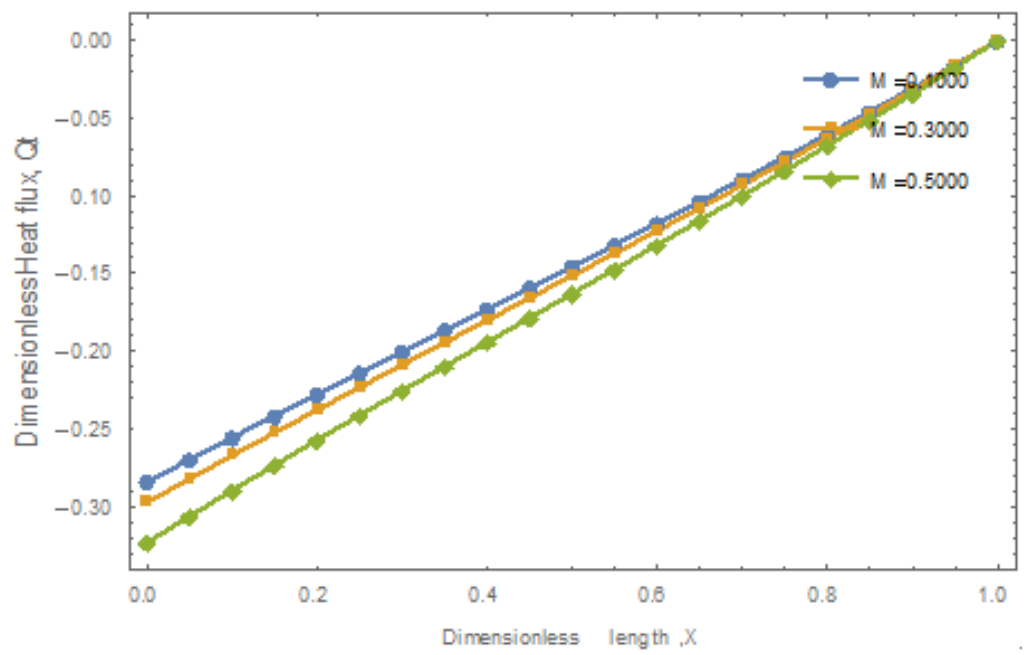

Figure 14. Effect of conductive-convective on the heat flux across fin length when $\beta=0.2 ; H_{t}=0.6 ; 0.5 ; G=0.4 ; P e=0.5$. 


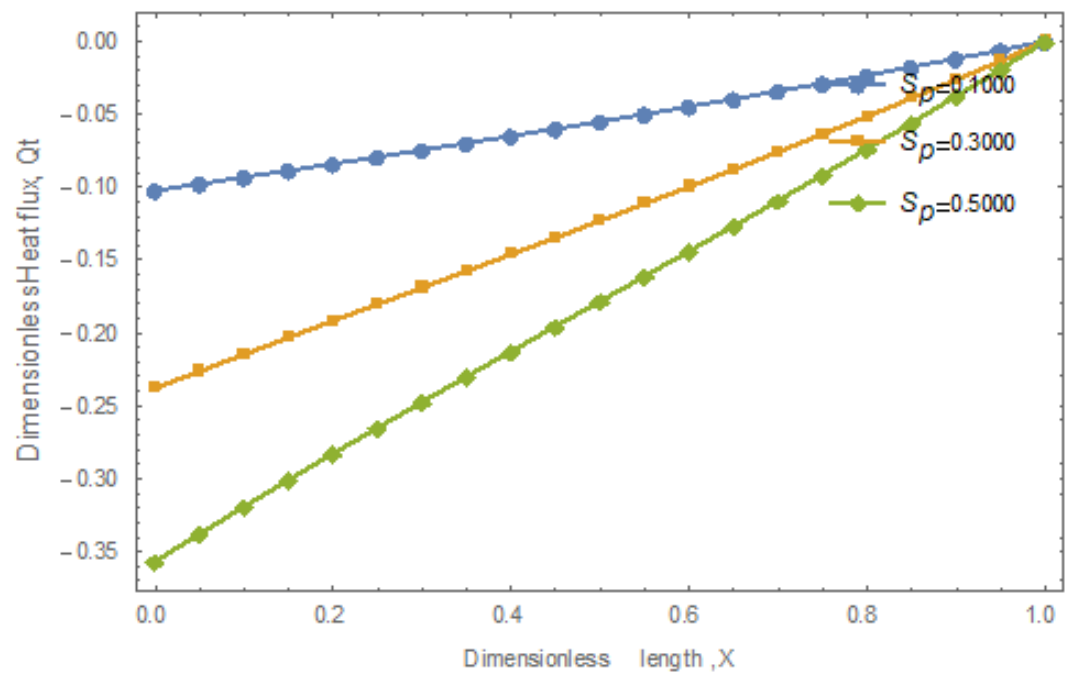

Figure 15. Effect of porosity on the Heat flux across fin length when $\beta=0.2 ; H_{t}=0.6 ; 0.5 ; G=0.4 ; P e=0.5$.

Figure 4 shows the effects of porous parameter or porosity on the temperature distribution in the porous fin are shown. From the figures, as the porosity parameter increases, the temperature decreases rapidly and the rate of heat transfer (the convective-radiative heat transfer) through the fin increases as the temperature in the fin drops faster (becomes steeper reflecting high base heat flow rates) as depicted in the figures. The rapid decrease in fin temperature due to increase in the porosity parameter is because as porosity parameter increases, the permeability of the porous fin increases and therefore the ability of the working fluid to penetrate through the fin pores increases, the effect of buoyancy force increases and thus the fin convects more heat, the rate of heat transfer from the fin is enhanced and the thermal performance of the fin is increased. High value of porosity parameter not only decreases the effective thermal conductivity but also reduces ideal heat transfer.

Figure 5 shows the effect on conductive-convective parameter on the temperature distribution along the length of a moving porous fin. From the figure below, it can be seen that the magnitude of the temperature is increased by decreasing the thermogeometric parameter $\mathrm{M}$. It is evident that as the convection-conduction parameter is increased it contributes to more heat loss from the fin and hence cooling of the fin occurs which shows a decrease in the temperature profile.

The effect of Peclet number on the thermal behaviour of the fin is shown in Figure 6. It is shown that that the fin temperature increases with an increase in the Peclet number. This is expected because with an increase in Peclet number, the material moves faster and the time for which the material is exposed to the environment gets shorter as well as the losing heat form fin surface gets stronger, thus the fin temperature increases. The influence of the thermal conductivity parameter $\beta$ on the temperature distribution along the fin is presented in Figure 7. It is clear from the figure that as the thermal conductivity parameter is increased the temperature distribution along the fin increases. Physically speaking, the effect of an increase in the thermal conductivity parameter enhances the heat conduction process and results in an increase in the local temperature of the fin. It is further observed that the fin-tip temperature increases with an increase in the thermal conductivity parameter. In order to get the best efficiency from the fin, the dimensionless thermal conductivity parameter $\beta$ should be kept as low as possible. Figure 7 shows the effect of internal heat generation on the temperature distribution in the fin. with a decrease in the internal heat generation number, the losing heat fin form surface gets stronger, thus the fin temperature decreases. Figures 8 and 9 shows the effect of generation parameter on the temperature distribution in the fin. As the generation parameter increases the fin temperature increases.

Figure 10 shows the impact of the nonlinear thermal conductivity and thermo-geometric parameters on the fin efficiency of the porous fin. The fin efficiency decreases monotonically (for different thermal conductivity) with increasing thermo geometric parameter. From the figures, it is shown that as the thermo geometric parameter increases, the efficiency of the fin decreases. It is also shown that as the conductive-convective value approaches a unity value, the fin efficiency becomes high as the effective thermal conductivity is reduced to a very small quantity.

Figure 11 illustrates the influence of nonlinear thermal conductivity parameter and Peclet number on the fin thermal efficiency of the porous fin. It is shown that the fin thermal efficiency increases as the Peclet increases. An increase in Peclet number indicates the speed of the fin material is high, if a material moves in high speed, the heat transfer rate is augmented, and hence efficiency is enhanced.

Figure 12 shows that the fin efficiency decreases with increase in internal heat generation. Fin efficiency is improved 
by increasing the peclet number.

Figures 13-15 shows the effects of thermal conductivity, convective and porosity parameters on the temperature gradient across the length of the fin. As expected, the temperature gradient decreases as the thermal conductivity parameter increases but increases as the porosity and convective parameter increases. But generally, heat flux increases across the length of the fin.

\section{Conclusion}

In this paper, the finite difference and differential Transformation methods have been applied for comparative study of thermal analysis of a moving convective porous fin with temperature dependent thermal conductivity and internal heat generation. The effects of the various parameters appearing in the thermal model on the temperature distribution, heat transfer rate and thermal efficiency of the longitudinal rectangularfin have been analyzed. From the analysis, it is found that increase in porosity, convective, increase the rate of heat transfer from the fin and consequently improve the efficiency of the fin while increase in thermal conductivity and internal heat generation decreases the rate of heat transfer from the fin. It is hope that the numerical and semi-analytical studies presented in this paper will help in providing good physical insights in practice.

\section{Nomenclature}

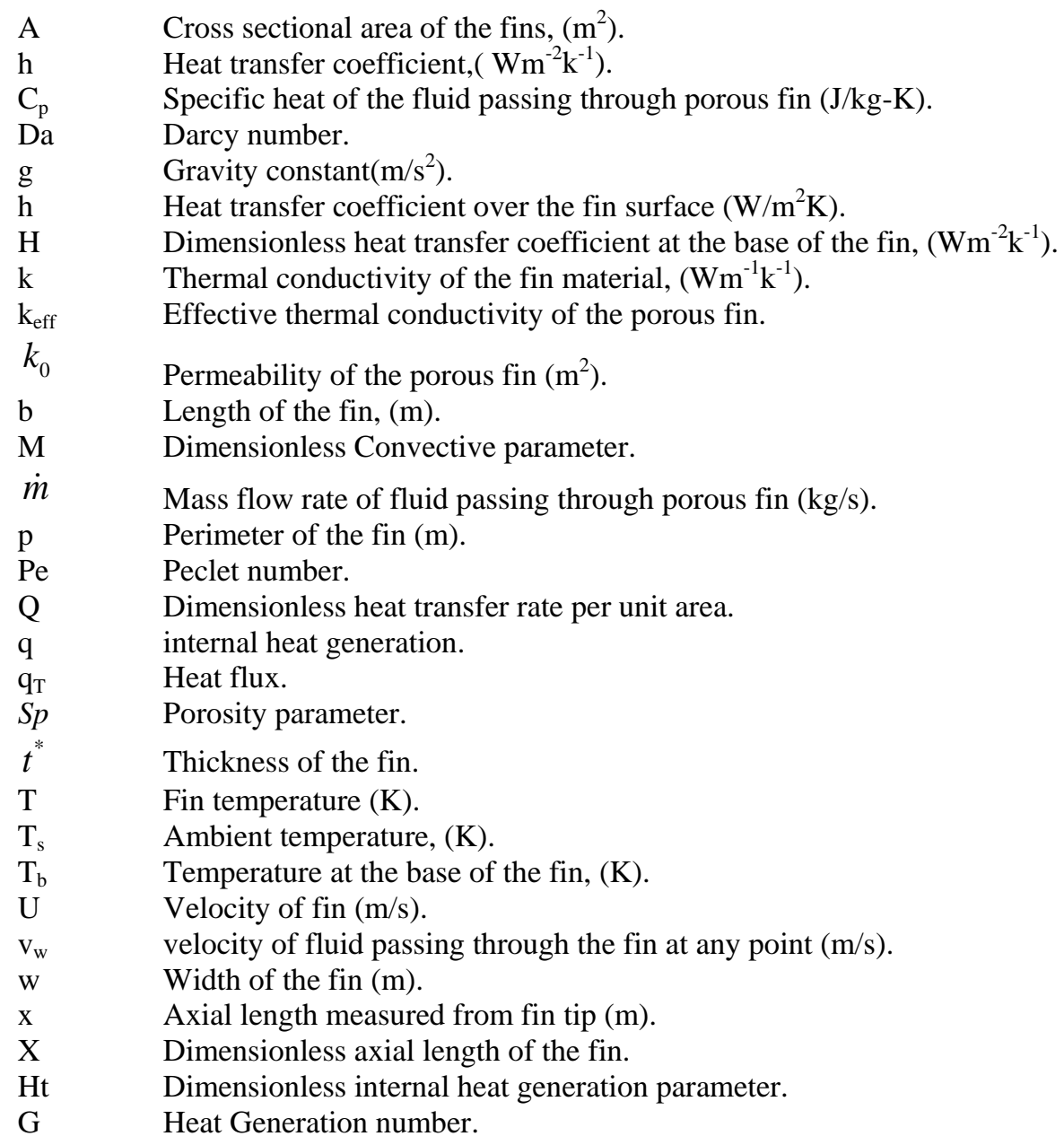

\section{Greek Symbols}

$\beta \quad$ Thermal conductivity parameter or non-linear parameter.

$\theta \quad$ Dimensionless temperature.

$\eta \quad$ Efficiency of the fin.

$v \quad$ Kinematic viscosity $\left(\mathrm{m}^{2} / \mathrm{s}\right)$. 
$\rho \quad$ Density of the fluid $\left(\mathrm{kg} / \mathrm{m}^{3}\right)$.

$\lambda \quad$ Measure of thermal conductivity variation with temperature.

\section{Subscripts}

$\begin{array}{ll}\mathrm{s} & \text { Solid properties. } \\ \mathrm{f} & \text { Fluid properties. } \\ \text { eff } & \text { Effective porous properties. }\end{array}$

\section{References}

[1] Kraus, A. D., Aziz, A., Welty, J. (2001). Extended surface heat transfer. Wiley; 2001.

[2] M. G. Sobamowo. (2016). Thermal analysis of longitudinal fin with temperature-dependent properties and internal heat generation using Galerkin's method of Weighted Residual. Applied Thermal Engineering, volume 99 2016, pp. 1316-1330.

[3] Chiu, C. H., Chen, C. K. (2002). A decomposition method for solving the convective longitudinal fins with variable thermal conductivity. Int J Heat Mass Transf, 2002, 45: 2067-2075.

[4] Arslanturk, C. (2005). A decomposition method for fin efficiency of convective straight fins with temperature-dependent thermal conductivity. Int Commun Heat Mass Transf, 2005, 32: 831-841.

[5] Rajabi, A. (2007). Homotopy perturbation method for fin efficiency of convective straight fins with temperature-dependent thermal conductivity. Phys Lett A, 2007, 364: 33-37.

[6] Domairry, G., Fazeli, M. (2009). Homotopy analysis method to determine the fin efficiency of convective straight fins with temperature-dependent thermal conductivity. Commun Nonlinear Sci Numer Simul, 2009, 14: 489-499.

[7] Kulkarni, D. B., Joglekar, M. M. (2009). Residue minimization technique to analyze the efficiency of convective straight fins having temperature-dependent thermal conductivity. Appl Math Comput, 2009, 215: 2184-2191.

[8] Bouaziz, M. N., Aziz, A. (2010). Simple and accurate solution for convective-radiative fin with temperature dependent thermal conductivity using double optimal linearization. Energ Convers Manage, 2010, 51: 2276-2782.

[9] Ranjan, D. (2011). A simplex search method for a conductive-convective fin with variable conductivity. Int J Heat Mass Transf, 2011, 54: 5001-5009.

[10] Aziz, A., Khani, F. (2011). Convection-radiation from a continuous moving fin of variable thermal conductivity. J Franklin Inst, 2011, 348: 640-651.

[11] Aziz, A., Lopez, R. J. (2011). Convection-radiation from a continuously moving, variable thermal conductivity sheet or rod undergoing thermal processing. Int J Therm Sci, 2011, 50: 1523-1531.

[12] Torabi, M., Yaghoobi, H., Aziz, A. (2012). Analytical solution for convective-radiative continuously moving fin with temperature-dependent thermal conductivity. Int J Thermophys, 2012, 33: 924-941.

[13] A. S. V. Ravi Kanth and N. Uday Kumar. Application of the Haar Wavelet Method on a Continuously Moving Convective-Radiative Fin with Variable Thermal Conductivity. Heat Transfer-Asian Research . DOI: 10.1002/htj.21038.

[14] Sharqawy, M. H., Zubair, S. M. (2008). Efficiency and optimization of straight fins with combined heat and mass transfer-an analytical solution. Appl Therm Eng, 2008, 28: 2279-2288.

[15] Fouladi, F., Hosseinzadeh, E., Barari, A., Domairry, G. (2010). Highly nonlinear temperature-dependent fin analysis by variational iteration method. Heat Transf Res, 2010, 41: 155-165.

[16] Malekzadeh, P., Rahideh, H., Karami, G. (2006). Optimization of convective-radiative fins by using differential quadrature method. Energy Convers Manag, 2006, 47: 1505-1514.

[17] Kundu, B., Aziz, A. (2010). Performance of a convectively heated rectangular fin with a step change in cross-sectional area and losing heat by simultaneous convection and radiation (step fins under radiation environment). J Heat Transf, 2010, 132: 104502-1.

[18] Ya-song Sun, Jing Ma. (2015). Application of Collocation Spectral Method to Solve a Convective-Radiative Longitudinal Fin with Temperature Dependent Internal Heat Generation, Thermal Conductivity and Heat Transfer Coefficient. Journal of Computational and Theoretical Nano-science, volume12, pp. 2851-2860.

[19] Mohsen Torabi, A. Aziz. (2012). Thermal performance and efficiency of convective- radiative T-shaped fins with temperature dependent thermal conductivity, heat transfer coefficient and surface emissivity. International Communications in Heat and Mass Transfer, volume 39, pp. 1018-1029.

[20] Darvishi, M. T., Gorla, R. S. R, Kani, F. (2013). Natural Convection and Radiation in Porous Fins. International Journal for Numerical Methods for Heat \& Fluid Flow, volume 23, pp. 1406-1420. 
[21] Abdelhalim, E. (2013). On A New Differential Transformation Method for Solving Nonlinear Differential Equation. Asian-European Journal of Mathematics, volume 6.

[22] Maheria, M. G. (2010). Thermal Analysis of Natural Convection and Radiation in Porous Fins. ETD Archive. Paper 447.

[23] Prasad, B. S. (1997). Fin Efficiency and Mechanisms of heat exchange through fins in multi-stream plate-fin heat exchanger: development and application of a rating algorithm. International Journal of Heat Transfer, volume 40, pp. 4279-4288.

[24] Singla, R. K. and Ranjan, D. (2014). Application of decomposition method and inverse parameters in a moving fin. Energy Conversion and Management, volume 84, pp. 268-281.

[25] J. K. Zhou. (1986). Differential Transformation method and its Application for electrical circuits. Hauzhang University Press, Wuhan (China), 1986.

[26] Moradi, A., Rafiee, R. (2013). Analytical Solution to Convection-Radiation of a Continuously Moving Fin with Temperature-Dependent thermal conductivity. Thermal Science, volume 17, pp. 1049-1060.

[27] Dogonchi, A. S., Ganji, D. D. (2016). Convection-Radiation heat transfer study of moving fin with temperature dependent thermal conductivity, heat transfer coefficient and heat generation. Applied Thermal Engineering, 103(2016), pp. 705-712.

[28] A. A. Joneidi, D. D. Ganji, M. Babaelahi. (2009). Differential Transformation Method to determine fin efficiency of convective straight fins with temperature dependent thermal conductivity. International Communications in Heat and Mass Transfer, volume 36, pp. 757-762.

[29] M. Torabi, H. Yaghoobi, and A. Aziz. (2012). Analytical Solution for Convective-Radiative Continuously Moving Fin with Temperature-Dependent Thermal Conductivity Int. J. Thermophysics, (2012), 33: 924-941.

[30] A. Aziz, Robert J. Lopez. (2011). Convection-radiation from a continuously moving, variable thermal conductivity sheet or rod undergoing thermal processing. I. J. of Thermal Sciences, 50(2011), 1523-1531.

[31] A. Aziz, F. Khani. (2011). Convection-radiation from a continuously moving fin of variable thermal conductivity. J. of Franklin Institute, 348(2011), 640-651.

[32] S. Singh, D. Kumar, K. N. Rai. (2013). Wavelet Collocation Solution for Convective-Radiative Continuously Moving Fin with Temperature-Dependent Thermal Conductivity. International Journal of Engineering and Advanced Technology (IJEAT), vol. 2(4), 2013.

[33] A. Aziz, M. Torabi. (2012). Covective-radiative fins with simultaneous variation of thermal conductivity, heat transfer coef ficient and surface emissivity with temperature. Heat transfer Asian Research, 41(2), (2012).

[34] J. Ma, Y. Sun, B. W. Li, H. Chen. (2016). Spectral collocation method for radiative-conductive porous fin with temperature dependent properties. Energy Conversion and Management, 111(2016), 279-288.

[35] Y. Sun, J. Ma, B. W. Li, H. (2015). Spectral collocation method for convective-radiative transfer of a moving rod with variable thermal conductivity. International Journal of Thermal Sciences, 90(2015), 187e196. 